\title{
Cardiac wall motion detection by neural network analysis on Tc-99m MIBI myocardial perfusion gated single photon emission computed tomography

\author{
Yu-Chien Shiau ${ }^{1,2}$, Hsiao-Hsuan Chou ${ }^{1}$, Shu-Hsun $\mathrm{Chu}^{3}$, Te-Son Kuo ${ }^{1}$ \\ 'Department of Electrical Engineering, National Taiwan University, TAIWAN \\ ${ }^{2}$ Department of Nuclear Medicine, and ${ }^{3}$ Cardiovascular Center, Far Eastem Memorial Hospital, TAIWAN
}

\begin{abstract}
In this study we investigated cardiac wall motion detection by back propagation neural network analysis on Tc-99m methoxyisobutylisonitrile (MIBI) myocardial perfusion gated single photon emission computed tomography (GSPECT). Serial doughnut-like phantom images were generated to simulate cardiac wall motion of left ventricle, and as training data. After the neural network was trained, the neural network can perform motion detection for all serial phantom images and also for all series of patients' GSPECT including short axis view, vertical long axis view, and horizontal long axis view images. The results of motion detection were displayed in the format of vector fields superimposed on the original GSPECT. The study showed that cardiac wall motion detection by back propagation neural network was useful in the evaluation of Tc-99m MIBI myocardial perfusion GSPECT.
\end{abstract}

Kegwords - neural network, motion detection, GSPECT

\section{INTRODUCTION}

Cardiac wall motion detection and analysis are very challenging and important for medical and cardiac image processing. Tc-99m methoxyisobutylisonitrile (MIBI) myocardial perfusion gated single photon emission computed tomography (GSPECT) [1] is useful in the study of myocardial perfusion and cardiac wall motion. Motion detection by neural networks is a relatively new method, and has become more successful in various simulation studies [2] and intended applications [3]. We investigate the methodology of motion detection by back propagation neural network applied to Tc-99m MIBI myocardial perfusion GSPECT for the analysis of cardiac wall motion.

\section{METHODOLOGY}

We used a three-layer back propagation neural network (Fig. 1) for cardiac wall motion detection. The brightness values of a $3 \times 3$ matrix in one image and another $3 \times 3$ matrix of the same location in the next image were selected as input data. There are totally 18 input data combined together to be an $18 \times 1$ column vector:

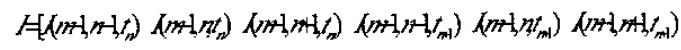

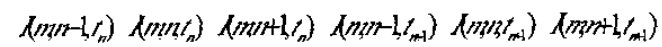 $\left.(n+1, n-1, n)\left(n+1, n r_{n}\right)(n+1, n+1, n)(n+1, n-1 / m)\left(n n+1 / t_{m}\right)(m+1, n+1 / m)\right)^{T}(1)$}

The hidden layer consisted of 14 nodes. The unit functions were logarithmic sigmoid transfer function. There were 9 output nodes. The first output node represents no motion between the two successive images. The remaining 8 nodes represented 8 directions of motion, namely moving toward the right $(1,0)$, the lower right $(1,1)$, downward $(0,1)$, the lower left $(-1,1)$, the left $(-1,0)$, the upper left $(-1,-1)$, upward $(0,-1)$, and the upper right $(1,-1)$. The training function was gradient descent momentum with an adaptive learning rate. The performance function was mean squared error performance function.

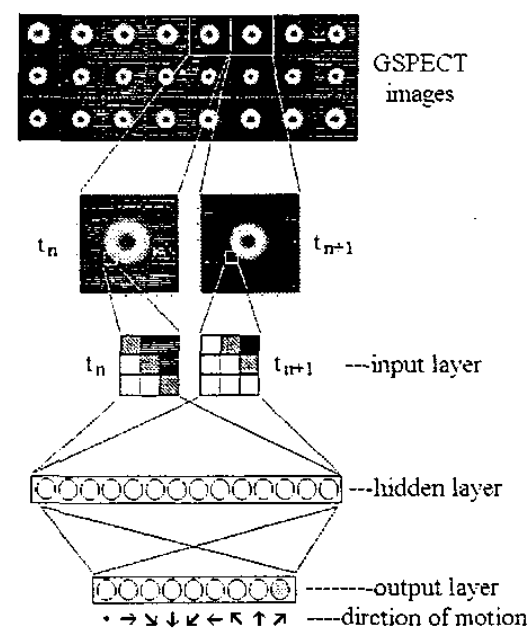

Fig. 1. Three-layer back propagation neural network for cardiac wall motion detection.

\section{A. Simulation of serial phantom images of the normal left ventricle}

Serial doughnut-like phantom images were generated to simulate the cardiac wall motion. The outer diameters (OD) of the serial phantom images were:

$$
O D(t)=18+3 \cos \left(\frac{2 \pi(t-1)}{24}\right) \text {, where } \mathrm{t}=1 \sim 24 \text {. }
$$

The image brightness function was

$$
E(x, y, h)=120\left(1-\cos \left(\frac{\left.2 \pi \sqrt{\left(x-x_{0}(t)\right)^{2}+\left(y-x_{11}(t)\right)^{2}}\right)}{O D}\right)\right. \text {, }
$$

where $\mathrm{x}_{0}(\mathrm{t})=31$, and $\mathrm{y}_{0}(\mathrm{t})=31$. The magnitudes of motion velocity between two successive images can be derived by

$$
r(x, y, t)=\frac{\pi}{4} \sin \left(\frac{2 \pi(t-1)}{24}\right) \frac{\sqrt{\left(x-r_{0}(f)\right)^{2}+\left(y-y_{0}(f)\right)^{2}}}{O D(t)} .
$$

The pixels with velocities $<0.25$ were regarded as no motion, and node 1 outputs were assumed. Velocities $>=$ 0.25 were rendered into the previously mentioned 8 directions, corresponding to the node 2 to node 9 of the outputs. We extracted total 4232 training data sets within the systolic and diastolic images. The neural network was trained until a convergence of 0.0001 was achieved. The neural network could thus perform motion detection for all of the 24 serial images.

\section{B. Simulation of serial phantom images with septal akinesia}

To simulate septal wall akinesia, the centers of the left ventricle were shifted by the formula

$$
x_{0}(t)=29+2 \cos \left(\frac{2 \pi(t-1)}{24}\right), y_{0}=31 .
$$

\section{Motion detection in parient GSPECT images}


Two patients received Tc-99m MIBI myocardial perfusion GSPECT. One patient had normal cardiac wall motion. The other patient had ischemic heart disease with akinesia to dyskinesia over the septal wall. Patients' images including short axis view, vertical long axis view, and horizontal long axis view were pre-processed with thresholding, filtering in spatial domain, and filtering in time domain with Gaussian filter. The images were fed to the trained neural network for motion detection. The outputs of the motion detection were displayed in the format of vector fields superimposed on the original images.

\section{RESULTS}

\section{A. Motion detection in phantom images of nommal left ventricle}

Motion detection in the serial phantom images simulating the normal left ventricle showed good performance. The accuracies of motion detection for the training data themselves were $100 \%$. The accuracies of motion detection for the entire series of phantom inages simulating the normal left ventricle averaged $95.4 \%$.

\section{B. Motion detection in phantom images with septal akinesia}

Motion detection on the serial phantom images simulating left ventricle with septal akinesia showed good performance and little motion over the akinetic septal wall.

\section{Motion derection in GSPECT images of the health" patient}

The results of motion detection by the neural network can reflect normal cardiac wall motion. The results were consistent with cardiac sonographic findings.
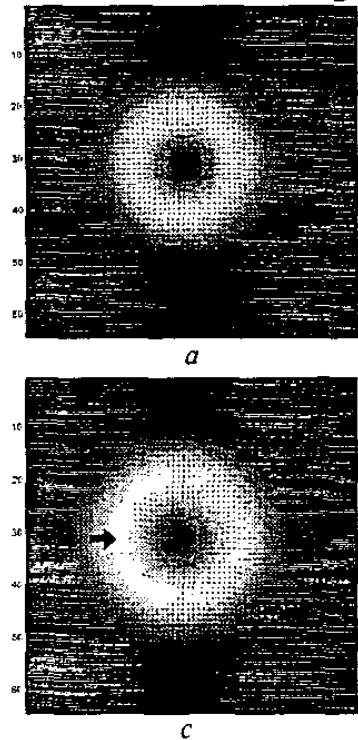

$c$
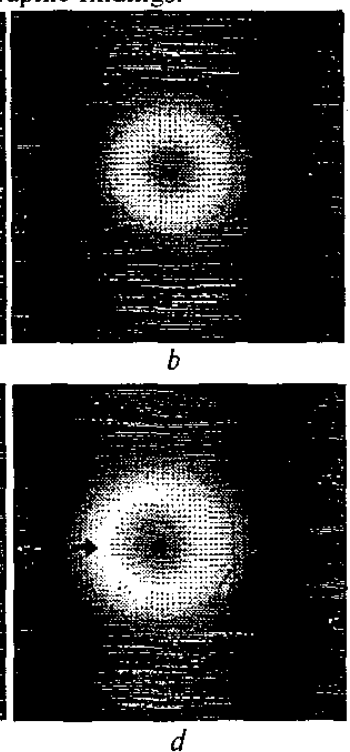

Fig. 2. Motion detection of the training data sets from images $t=5 \sim 6$ and $t=17 \sim 18$ (b). Akinesia can be observed over septal wall (anrows) in systolic phase (c) and diastolic phase (d).
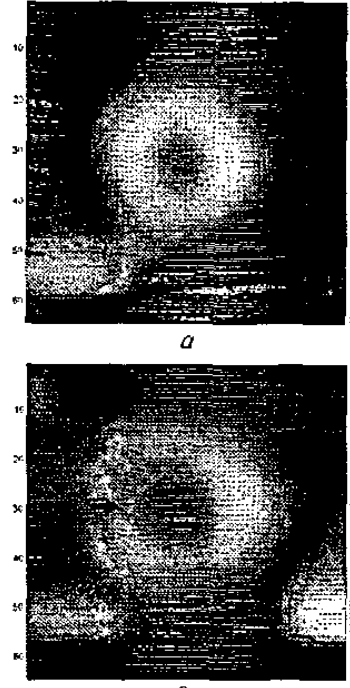

c

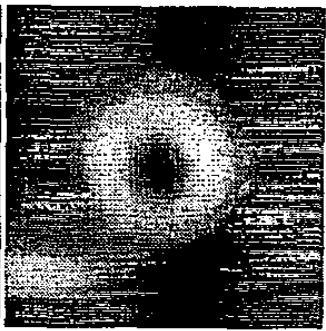

$b$

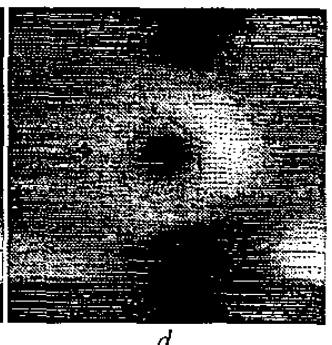

d
Fig. 3. Motion delection in normal patien's GSPECT showed norma cardiac wall motion in shor axis view (a. b). Dyskinesia can be observed over patient's septal wall (arrow) (c, d).

\section{Motion detection in GSPECT images with septal dyskinesia}

The motion detection showed dyskinesia over the septal wall.

\section{DISCUSSION and CONCLUSION}

The selection of training data for back propagation neural network is important for its perfomance. The preprocessing of patients' GSPECT images is also important for motion detection by back propagation neural network. The motion detection by the neural network for patients' GSPECT images can detect normal cardiac wall motion, and also detect the dyskinesia of patients' abnormal cardiac wall motion. The results were consistent with the findings of cardiac sonography. The study showed that cardiac wall motion detection by back propagation neural network was useful in the evaluation of Tc-99m MIBI GSPECT inages.

\section{ACKNOWLEDGMENT}

The authors thank Far Eastern Memorial Hospital, Taiwan for the research grant FEMH-A900010.

\section{REFERENCES}

[1] J.P. Ioannidis, T.A. Trikalinos, P.G. Danias, "Electrocardiogram-gated single-photon emission computed tomography versus cardiac magnetic resonance imaging for the assessment of left ventricular volumes and ejection fraction: a meta-analysis," Joumal of the American College of Cardiology; vol. 39, pp. 2059-2068, 2002.

[2] E. Atsumi, M. Takagi, and K. Yokosawa, "Local motion detection by hierarchical neural network," Slstems and Computers in Japan, vol. 25, No. 11, pp. 24-35, 1994.

[3] T. Roska, T. Boros, A. Radvanyi, P. Thiran, and L.O. Chua, "Detecting moving and standing objects using cellular neural networks," International Journal of circuit theory and applications, vol. 20, pp. 613-628, 1992. 